\title{
Vertebroplasty Increases the Incidence Of New Vertebral Compression Fractures Compared To Conservative Management
}

\author{
Maram Othman 1,* Meshal Alshaalan",* Abdulrahman Khawaji ${ }^{1} \quad$ Rawan Benkuddah ${ }^{1}$ \\ Hala Khali ${ }^{2} \quad$ Khalid Alismail ${ }^{1} \quad$ Waleed Althobaity ${ }^{1}$ Saleh Alreshoodi ${ }^{1}$
}

\author{
${ }^{1}$ Medical Imaging Department, King Faisal Specialist Hospital and \\ Research Centre, Riyadh, Kingdom of Saudi Arabia \\ ${ }^{2}$ Department of Biostatistics, Epidemiology and Scientific \\ Computing, King Faisal Specialist Hospital \& Research Centre, \\ Riyadh, Kingdom of Saudi Arabia
}

\begin{abstract}
Address for correspondence Maram Al Othman, MD, Medical Imaging Department, King Faisal Specialist Hospital and Research Centre, Riyadh, Kingdom of Saudi Arabia, (e-mail: Alothman.maram@gmail.com).
\end{abstract}

\begin{abstract}
Background Vertebral osteoporotic fractures are a major cause of morbidity and disability among the elderly population.

This study sought to compare the incidence of new vertebral fractures in patients treated by vertebroplasty with that of those managed conservatively, while also assessing the potential risk factors contributing to the occurrence of these fractures.

Materials and Methods The details of a total of 121 eligible subjects with radiologically proven osteoporotic vertebral fractures were retrieved from our archive between January 2010 and September 2019 and divided, based on the treatment method, into percutaneous vertebroplasty (PVP) $(n=60)$ and nonsurgical treatment $(n=61)$. The included subjects' clinical data, demographic profiles, and imaging findings on plain radiography, CT, and MRI scans performed at baseline and within 24 months following treatment were reviewed and documented.

Results The difference in the incidence of new fractures was statistically significant $(p=0.001)$, with rates of $70 \%(n=32)$ in the vertebroplasty group and $30 \%(n=14)$ in the conservatively treated cases reported at a median follow-up time point of approximately 4 months. The presence of liver disease $(p=0.037)$, a history of transplantation $(p=0.003)$, the use of steroids $(p=0.023)$, a low-bone mineral density (BMD) score $(p=0.023)$, and a higher number of fractures on baseline imaging $(p=0.013)$ were associated with a greater risk of subsequent vertebral fractures.

Keywords Conclusions The incidence of acute fractures after PVP is higher than that among patients treated conservatively. Decision-making regarding the adoption of this intervention should take into consideration the risk factors leading to a greater risk of subsequent vertebral fractures, such as a higher number of fractures at baseline imaging, low-BMD score, patient comorbidities, and steroid use.
\end{abstract}

"Joint first authors.

DOI https://doi.org/ $10.1055 / \mathrm{s}-0041-1731603$ ISSN 2542-7075 (c) 2021. The Pan Arab Interventional Radiology Society

This is an open access article published by Thieme under the terms of the Creative Commons Attribution-NonDerivative-NonCommercial-License, permitting copying and reproduction so long as the original work is given appropriate credit. Contents may not be used for commercial purposes, or adapted, remixed, transformed or built upon. (https://creativecommons.org/licenses/by-nc-nd/4.0/). Thieme Medical and Scientific Publishers Private Ltd. A-12, Second Floor, Sector -2, NOIDA -201301, India 


\section{Background}

The occurrence of new vertebral fractures is a known complication that may appear following vertebral augmentation, particularly in areas adjacent to the treated level in a manner attributed to the increased stiffness of the cemented vertebra, which alters vertebral biomechanics, transferring the load to the nonaugmented adjacent level and increasing the risk for fracture to approximately $12 \%$ at 2 years and $52 \%$ at 4 years. ${ }^{1-5}$

Even though the role of vertebroplasty in facilitating a rapid reduction in pain, and an earlier return to daily life activities is a widely acceptable fact, significant controversy in the literature persists regarding whether vertebral augmentation increases the risk of vertebral fractures. ${ }^{6-11}$

The present study aimed to determine the incidence of new osteoporotic spinal fractures in patients treated by vertebroplasty versus conservative treatment. It also sought to evaluate potential risk factors contributing to the occurrence of these fractures following vertebroplasty. The outcomes of this study may assist physicians in choosing the best treatment regimen for their patients.

\section{Material and Methods}

The Research Ethics Committee at KFSHRC Riyadh, Saudi Arabia, approved this retrospective study and waived the requirement to collect informed consent. We retrospectively revised the data of patients who suffered from radiologically proven acute osteoporotic vertebral compression fractures-93 of whom underwent vertebroplasty and 361 of whom were treated conservatively-who were then followed-up with by plain radiography or cross-sectional imaging at KFSHRC between January 2010 and September 2019. The study exclusion criteria included an absence of posttreatment imaging follow-up and the existence of vertebral fractures caused by malignancy or major trauma. Thirty-three patients who underwent vertebroplasty and 300 patients who were treated conservatively were excluded, based on these exclusion criteria.

The included subjects' clinical data, demographic profiles, and imaging findings on plain radiography, CT, and MRI scans performed at baseline and within 24 months following treatment were reviewed and documented.

The median ages of the conservatively managed patients and those treated with the percutaneous vertebroplasty (PVP) protocol were 64 years and 67 years, respectively. There were significantly more patients with liver disease $(n=23 / 60)$, a history of liver transplant $(n=25 / 60)$, and those using steroids $(n=32 / 60)$ in the vertebroplasty group. We also recorded a significantly lower mean bone mineral density (BMD) score among patients who underwent vertebroplasty $(p<0.001)$. Only 12 subjects from nonsurgical treatment (NST) group and 18 subjects from the vertebroplasty group were on antiresorptive medication. Meanwhile, patients' sex and age did not statistically correlate with the risk of subsequent new vertebral fractures. The characteristics of both groups are listed in - Table 1 .

Acute vertebral osteoporotic fractures were defined as occurrences of newly developed spinal pain on palpation, with imaging evidence of acute vertebral collapse/fracture line on various modalities or on MRI as bone marrow edema-like signals, corresponding to the compressed trabecula with or without significant end-plate depression or height loss.

The location and number of fractures at baseline and follow-up, the levels managed by vertebral augmentation procedures, the frequency of vertebroplasty/kyphoplasty sessions, and related complications were assessed.

Table 1 Summary of all included patients' characteristics

\begin{tabular}{|c|c|c|c|c|}
\hline & NST $(n=61)$ & $\operatorname{PVP}(n=60)$ & Total $(n=121)$ & $p$-value ${ }^{a}$ \\
\hline & \multicolumn{3}{|l|}{$n(\%)$} & \\
\hline Male & $27(44)$ & $23(38)$ & $50(41)$ & 0.508 \\
\hline Liver disease & $11(32)$ & $23(68)$ & $34(28)$ & 0.013 \\
\hline Renal disease & $2(29)$ & $5(71)$ & $7(5.8)$ & 0.234 \\
\hline History of transplant & $11(31)$ & $25(69)$ & $36(30)$ & 0.004 \\
\hline Fractures at FU & $14(30)$ & $32(70)$ & $46(38)$ & 0.001 \\
\hline Antiresorptive medications & $12(40)$ & $18(60)$ & $30(25)$ & 0.188 \\
\hline Vitamin $\mathrm{D} / \mathrm{Ca}^{2+}$ & $34(44)$ & $44(56)$ & $78(64.5)$ & 0.043 \\
\hline \multirow[t]{2}{*}{ Steroids } & $19(37)$ & $32(63)$ & $51(42)$ & 0.023 \\
\hline & \multicolumn{3}{|c|}{$($ Mean \pm SD) } & \\
\hline \multirow[t]{2}{*}{ BMD } & $2.18 \pm 1.21$ & $3.03 \pm 0.99$ & $2.62 \pm 1.18$ & $<0.001^{b}$ \\
\hline & \multicolumn{3}{|c|}{ Median (min-max) } & \\
\hline Age (years) & $64(16-90)$ & $67.5(16-90)$ & $66(16-90)$ & $0.156^{c}$ \\
\hline
\end{tabular}

Abbreviations: BMD, bone mineral density; FU, follow-up; NST, nonsurgical treatment; PVP, percutaneous vertebroplasty.

a Pearson's Chi-square test

${ }^{\mathrm{b}} \mathrm{p}$-value derived from Student's $t$-test analysis

c $p$-value derived from Mann-Whitney $U$ test 
Vertebral augmentation procedures in our institution are performed by board-certified musculoskeletal radiologists. Conventional vertebroplasty is the most frequently performed intervention, with kyphoplasty and implant-assisted vertebroplasty adopted in a few selected cases.

Patients are considered to be eligible for such interventions if they present with severe spinal pain, aggravated by palpation of the affected levels without radicular symptoms, and fail to respond to conservative treatment.

\section{Vertebroplasty}

All our procedures are conducted with aseptic techniques and general anesthesia. Eleven or 13-gauge coaxial vertebroplasty needle is advanced, using a costovertebral or transpedicular approach, with the needle tip positioned within the anterior two-thirds of the vertebral body on the lateral projection. Subsequently, the polymethyl methacrylate (PMMA) mixture is injected under continuous fluoroscopic monitoring, with the operator watching for any cement leakage. After achieving adequate cement distribution, the needles are carefully withdrawn. All patients are instructed to follow the standard postprocedure precautions.

\section{Statistics}

Descriptive statistical analyses were conducted for baseline and demographic characteristics, adopting mean and standard deviation or median values for continuous variables. Intergroup comparisons were performed using the Chi-squared test for categorical variables or a parametric $t$-test or Mann-Whitney U test for continuous variables.

Single and multiple logistic regression analyses were conducted to examine the type of treatment as a predictor of fracture occurrence at follow-up.

We used the Kaplan-Meier (KM) curve to describe the rate of acute fracture over the observation period. For comparing KM curves, we used the log-rank test (Mantel-Haenszel) to assess differences between the two treatment groups.

A $p$-value of less than 0.05 was considered to be statistically significant.

The statistical analyses were performed using the Statistical Package for the Social Sciences for Windows version 25 software program (IBM Corporation, Armonk, NY, USA).

\section{Results}

A total of 121 cases with acute osteoporotic compression fractures were included in our study, divided into two main groups of patients who underwent vertebroplasty $(n=60)$ or were treated conservatively $(n=61)$. Three patients who were treated conservatively presented to our emergency department with recurrent acute vertebral fractures after a period of six to 12 months and were treated nonsurgically as well. Moreover, three patients from the vertebroplasty group underwent a second vertebroplasty session, and one patient underwent three separate sessions for additional acute fractures.
The incidence rates of acute vertebral fractures were $70 \%$ $(n=32)$ in the vertebroplasty group and $30 \%(n=14)$ in the conservatively treated group; thus, there was a statistically significant difference noted between the groups regarding the incidence rate $(p=0.001)$. This finding was also established by assessing the KM curves. Specifically, the NST group was at a lower risk for the development of new fractures during follow-up as compared with the PVP group $(p=0.024, \log$-rank test). Subsequent acute fractures occurred in both groups within a similar median follow-up period of $4.2(0.4-20.1)$ months.

Most of the vertebral fractures observed had developed in a single vertebra in both groups, accounting for $71.4 \%$ ( $n=10 / 14)$ among the conservatively managed patients and $71.9 \%(n=23 / 32)$ among the vertebroplasty cases. The maximum number of acutely fractured vertebrae seen during a single follow-up appointment was five vertebrae in both groups. There was a positive trend for the probability of subsequent vertebral fractures in relation to the number of acute fractures at baseline noted $(p=0.013)$. Our findings were confirmed using a simple and multiple logistic regression analysis of the relationship between acute fractures during follow-up and potential risk factors as shown in - Tables 2 and 3.

The location and number of vertebral fractures at initial and follow-up imaging in both groups are displayed in

-Fig. 1. Both adjacent and nonadjacent levels to the fractured

Table 2 Simple logistic regression of fracture presence at FU in relation to potential risk factors $(n=121)$

\begin{tabular}{|l|l|l|}
\hline \multicolumn{2}{|l|}{ OR $(95 \% \mathrm{Cl})$} & p-value \\
\hline Treatment groups & \multicolumn{2}{|l|}{} \\
\hline NST & Reference & \\
\hline PVP & $3.84(1.75-8.40)$ & $\mathbf{0 . 0 0 1}$ \\
\hline Age & $0.98(0.96-1.01)$ & 0.189 \\
\hline Gender & \multicolumn{2}{|l|}{} \\
\hline Male & Reference & \\
\hline Female & $1.34(0.63-2.85)$ & 0.45 \\
\hline Baseline no. of acute fractures & $1.44(1.08-1.93)$ & $\mathbf{0 . 0 1 3}$ \\
\hline Baseline BMD & $1.53(1.06-2.20)$ & $\mathbf{0 . 0 2 3}$ \\
\hline Transplant & \multicolumn{2}{|l|}{} \\
\hline Yes & Reference & \\
\hline No & $0.30(0.13-0.67)$ & $\mathbf{0 . 0 0 3}$ \\
\hline Liver disease & \multicolumn{2}{|l}{} \\
\hline Yes & Reference & \\
\hline No & $0.42(0.19-0.95)$ & $\mathbf{0 . 0 3 7}$ \\
\hline Renal disease & \multicolumn{2}{|l}{} \\
\hline Yes & Reference & 0.082 \\
\hline No & 0.23 \\
& $(0.042-1.21)$ & \\
\hline
\end{tabular}

Abbreviations: $\mathrm{BMD}$, bone mineral density; $\mathrm{Cl}$, confidence interval; $\mathrm{FU}$, follow-up; NST, nonsurgical treatment; OR, odds ratio; PVP, percutaneous vertebroplasty. 
Table 3 Multiple logistic regression of fracture presence at $\mathrm{FU}$ in relation to potential risk factors $(n=121)$

\begin{tabular}{|l|l|l|}
\hline & OR $(95 \% \mathrm{Cl})$ & $\boldsymbol{p}$-value \\
\hline Treatment groups & & \\
\hline NST & Reference & \\
\hline PVP & $3.56(1.28-9.87)$ & $\mathbf{0 . 0 1 5}$ \\
\hline Age & $0.97(0.93-1.01)$ & 0.093 \\
\hline Gender & & \\
\hline Male & Reference & \\
\hline Female & $3.02(0.99-9.22)$ & 0.052 \\
\hline Baseline no. of acute fractures & $1.09(0.77-1.55)$ & 0.612 \\
\hline Baseline BMD & $1.14(0.72-1.80)$ & 0.581 \\
\hline Transplant & & \\
\hline Yes & Reference & \\
\hline No & $0.17(0.02-1.81)$ & 0.14 \\
\hline Liver disease & & \\
\hline Yes & Reference & \\
\hline No & $\begin{array}{l}1.88 \\
0.18-19.68)\end{array}$ & 0.598 \\
\hline
\end{tabular}

Abbreviations: $\mathrm{BMD}$, bone mineral density; $\mathrm{Cl}$, confidence interval; $\mathrm{FU}$, follow-up; NST, nonsurgical treatment; OR, odds ratio; PVP, percutaneous vertebroplasty.

Note: Adjusted for treatment group, age, sex, baseline no. of acute fractures, baseline BMD, transplant, liver disease

vertebrae were involved; however, adjacent fractures represented the predominant finding in both groups. Twenty of 32 fractures (62.5\%) occurred immediately adjacent to the original vertebral fracture after PVP as compared with nine of 14 adjacent fractures (64.3\%) in the conservative treatment group. Examples from imaging follow-up of two cases are presented in - Figs. 2 and $\mathbf{3}$.

Standard vertebroplasty, as described in our methodology, was performed in all the cases included in this study, except for in three patients who underwent implant-assisted vertebroplasty. The maximum number of treated vertebra in a single session was four vertebrae. The treated levels were located between D5 and S1, with L1 constituting the most frequently cemented vertebra.

One patient who underwent vertebroplasty for a D11 vertebral fracture developed cord compression 3 months after the procedure, which was attributed to combined disc herniation and cement leakage into the D11-12 disc as shown in -Fig. 4. This patient experienced severe pain related to the

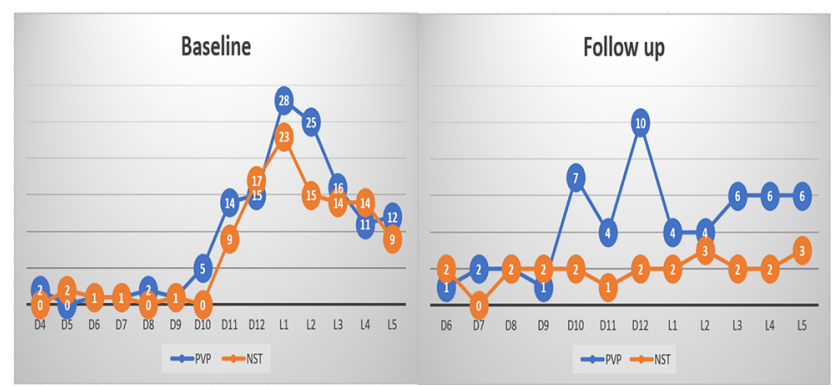

Fig. 1 Location and number of vertebral fractures

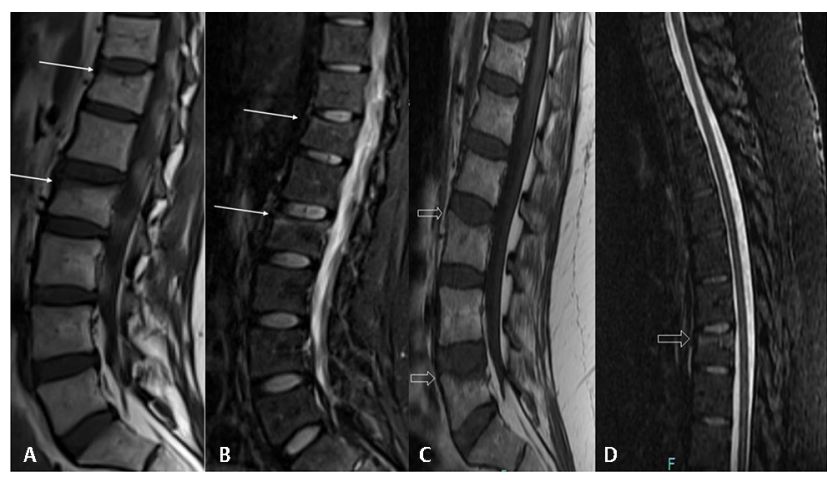

Fig. 2 Case 1. 31-year-old female known case of rheumatoid arthritis presented with acute vertebral fractures at D12 and L2 vertebra. $\operatorname{MRI}(\mathbf{a}, \mathbf{b})$ at baseline show superior end plates compression fractures involving D12 and L2 vertebrae (arrows), with corresponding bone marrow edema on T1-weighted image (T1WI) and short tau inversion recovery (STIR), a newly developed superior end plate compression fractures noted at D8, L3, L5 vertebrae (open arrows) on follow-up MRI (c, d).

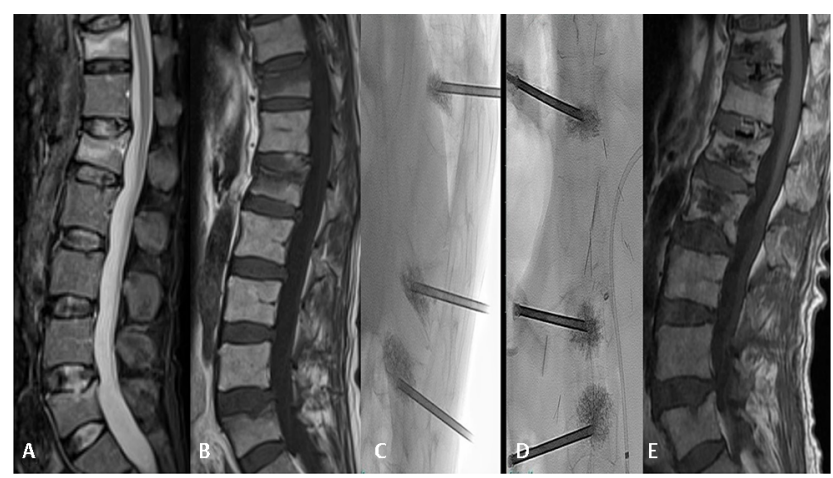

Fig. 3 Case 2. 61-year-old male status postliver transplant complained of sever thoracolumbar pain. Baseline MRI (a, b) shows superior end plates compression fractures involving D11 and L1 vertebrae (arrows), lateral and frontal projections obtained postvertebroplasty (c, d) show the unilateral left transpedicular approach, with adequate cement distribution within the treated levels, a superior end plate depression on T1-weighted image (T1WI) seen at the sandwiched D12 vertebra on follow-up MRI (e).

new adjacent fractures; however, he did not suffer from any focal neurological deficits and was treated conservatively. The remaining procedures were successfully accomplished without major complications. Nevertheless, minor technical complications in the form of cement leakage into the intervertebral discs in 15 of 60 (25\%) sessions or the paravertebral veins in five of 60 (8.3\%) sessions were observed. The analysis of these procedural complications revealed no significant association with the occurrence of subsequent new fractures.

\section{Discussion}

Vertebral augmentation techniques are mostly deployed in patients who have failed to show a benefit with conservative management. Their main advantages include the provision of immediate pain relief, stabilization of the fracture, and avoidance of the complications associated with strict bed rest. Yuan et al reviewed 10 randomized controlled trials (RCTs) 


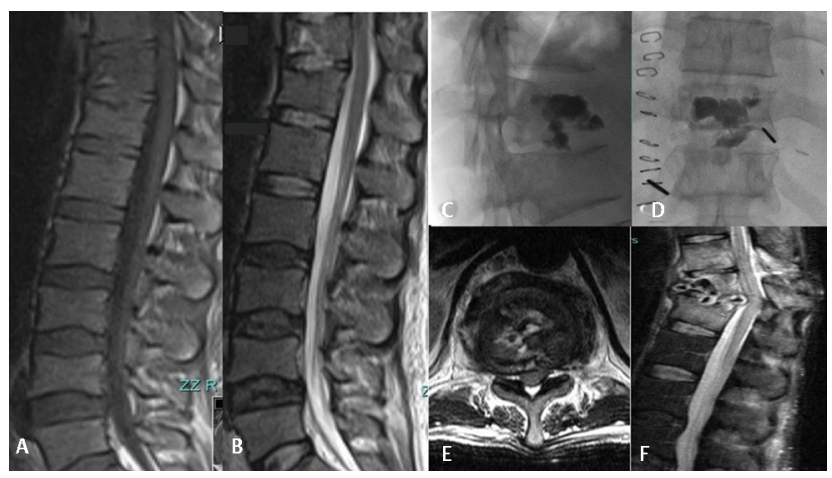

Fig. 4 Case 3. 50-year-old male status postliver transplant presented with acute midthoracolumbar. Baseline MRI $(\mathbf{a}, \mathbf{b})$ shows D11 anterior wedging and marrow edema on T1-weighted image (T1WI) and short tau inversion recovery (STIR), lateral and frontal projections obtained postvertebroplasty $(\mathbf{c}, \mathbf{d})$ display extrusion of polymethyl methacrylate (PMMA) injectate into the inferior D11-12 disc space, the subsequent MRI (e, f) demonstrate progressive D11 vertebral collapse with the leaked cement and the parent disc material herniate into the epidural space, resulting in moderate spinal canal stenosis on axial T2-weighted image (T2WI), additional adjacent acute compression fractures of both D10 and D12 are noted on STIR, causing focal kyphosis as well.

involving a total of 626 patients managed with vertebral augmentation and 528 patients treated conservatively and found that vertebroplasty/kyphoplasty resulted in a greater degree of pain relief and higher quality of life as compared with NST. ${ }^{12}$

The merit of vertebroplasty is still being questioned. The occurrence of new vertebral fractures following vertebroplasty is one of the most common complications, which has a negative impact on the therapeutic goal of these procedures. These fractures can be recurrent, adjacent, or nonadjacent to the treated level. ${ }^{13}$

Structural spinal alterations as a result of vertebral compression fractures generally increase the incidence of new vertebral body fractures by $19.2 \%$ within the first year of diagnosis, as reported by Lidsay et al. ${ }^{14,15}$

PMMA injection into the fractured vertebrae during vertebral augmentation is presumed to reduce the compliance of this vertebra, which would alter the biomechanics of load transfer, positioning the remaining vertebrae at greater risk of collapse. ${ }^{16}$ Polikeit et al stated that cement augmentation assists with increasing the strength of the fractured vertebra; yet it places a greater load on the adjacent vertebrae. ${ }^{17}$

Many studies in the literature have investigated whether vertebral augmentation procedures are correlated with higher rates of new vertebral fractures as compared with NST. A meta-analysis published in 2017 by Zhang et al, who assessed a total of 12 RCTs and prospective studies, reported no increase existed in the incidence of adjacent-level fractures following vertebroplasty/kyphoplasty as compared with after NST. ${ }^{18}$ Another study conducted by Trout et al determined that vertebral bodies adjacent to those treated with vertebroplasty experience a greater risk for fractures, typically occurring sooner than in nonadjacent ones. ${ }^{13}$
Nevertheless, the results of our study have revealed that the incidence of subsequent fractures was significantly higher among those who underwent vertebral augmentation relative to patients who received NST.

Lindsay et al performed an observational study including 381 women who sustained osteoporotic vertebral fractures and stated that the presence of prevalent vertebral fractures significantly enhanced the risk after an incident fracture from $4 \%$ in those with no fractures to $24 \%$ in those with two or more fractures. ${ }^{14}$ In this study, we also observed a significant relationship between the number of acute fractures at baseline and the risk of new fractures at follow-up. This might explain the higher incidence of subsequent fractures in our PVP patients, in addition to their significantly lower BMD score, greater use of steroids, and co-existence of comorbidities.

Several other risk factors have been also linked to vertebral fractures following vertebral augmentation, such as older patient age, low body mass index (BMI), and intradiscal cement leak. ${ }^{19}$ Additional vertebroplasty complications are also related to the extravasation of cement outside the fractured vertebra into the adjacent intervertebral disc, epidural space, and anterior venous plexus. The incidence of asymptomatic extravasation is $20 \%$ to $30 \%$ versus $0.1 \%$ for a symptomatic or serious leak. ${ }^{20}$

A recent prospective RCT by Yang et al and several other publications have emphasized the role of vertebroplasty as a successful method for achieving adequate pain relief and improved functional outcomes. ${ }^{21-23}$ Some of our cases were assessed with a visual analog pain score; yet the lack of standardized methods by which to document pain scores as well as the functional status in either group precluded us from including this as a measurable outcome in our study.

Our study has several limitations. The most important of all is the fact that it was a retrospective review of medical charts and imaging studies. Another issue was the lack of clinical data regarding patient compliance with antiresorptive medication, due to inconsistent documentation of these factors in the records. Conducting further prospective studies and RCTs specifically focused on the comparative incidence of vertebral fractures and associated risk factors would have a significant impact on the decision to perform vertebroplasty procedures, especially in elderly patients with multiple comorbidities and posttransplant patients.

\section{Conclusion}

There was an increased incidence of acute fractures recorded after percutaneous vertebroplasty as compared with in patients treated conservatively. Although vertebroplasty is considered to be a relatively safe, minimally invasive procedure, conducting such an intervention in our patients should be tailored to individual cases, based on standard appropriateness criteria and should take into consideration the risk factors associated with a heightened risk of subsequent vertebral fractures such as a greater number of fractures at baseline imaging, a low BMD score, patient comorbidities, and steroid use. 


\section{Ethics Approval and Consent to Participate}

Ethical approval for the study was obtained from Research Ethics Committee (REC) at KFSHRC in Riyadh.

\section{Consent for Publication \\ Not applicable}

\section{Availability of Data and Material}

The datasets used and/or analyzed during the current study are available from the corresponding author on reasonable request.

\section{Competing Interests}

The authors declare that they have no competing interests.

\section{Funding}

No funding were received for the current study.

\author{
Authors' Contributions \\ Study conception and design: M.A, M.S. \\ Acquisition of data: A.K, R.B. \\ Analysis and interpretation of data: H.K, K.I, W.T. \\ Drafting of manuscript: M.A, M.S. \\ Critical revision: S.R.
}

\section{Conflict of Interest}

None declared.

\section{Acknowledgments}

I would like to express my special thanks of gratitude to Mr. Fahad Alderwish, clinical application specialist. for retrieving the required studies from the PACS.

\section{References}

1 Grados F, Depriester C, Cayrolle G, Hardy N, Deramond H, Fardellone P. Long-term observations of vertebral osteoporotic fractures treated by percutaneous vertebroplasty. Rheumatology (Oxford) 2000;39(12):1410-1414

2 Lin EP, Ekholm S, Hiwatashi A, Westesson PL. Vertebroplasty: cement leakage into the disc increases the risk of new fracture of adjacent vertebral body. AJNR Am J Neuroradiol 2004;25(2):175-180

3 Uppin AA, Hirsch JA, Centenera LV, Pfiefer BA, Pazianos AG, Choi IS. Occurrence of new vertebral body fracture after percutaneous vertebroplasty in patients with osteoporosis. Radiology 2003;226(1):119-124

4 Berlemann U, Ferguson SJ, Nolte LP, Heini PF. Adjacent vertebral failure after vertebroplasty. A biomechanical investigation. J Bone Joint Surg Br 2002;84(5):748-752

5 Lee WS, Sung KH, Jeong HT, et al. Risk factors of developing new symptomatic vertebral compression fractures after percutaneous vertebroplasty in osteoporotic patients. Eur Spine J 2006;15(12):1777-1783

6 Farrokhi MR, Alibai E, Maghami Z. Randomized controlled trial of percutaneous vertebroplasty versus optimal medical management for the relief of pain and disability in acute osteoporotic vertebral compression fractures. J Neurosurg Spine 2011;14(5):561-569

7 Diamond TH, Bryant C, Browne L, Clark WA. Clinical outcomes after acute osteoporotic vertebral fractures: a 2-year non-randomised trial comparing percutaneous vertebroplasty with conservative therapy. Med J Aust 2006;184(3):113-117
8 Wang $\mathrm{HK}$, Lu K, Liang CL, et al. Comparing clinical outcomes following percutaneous vertebroplasty with conservative therapy for acute osteoporotic vertebral compression fractures. Pain Med 2010;11(11):1659-1665

9 Movrin I. Adjacent level fracture after osteoporotic vertebral compression fracture: a nonrandomized prospective study comparing balloon kyphoplasty with conservative therapy. Wien Klin Wochenschr 2012;124(9-10):304-311

10 Diamond TH, Champion B, Clark WA. Management of acute osteoporotic vertebral fractures: a nonrandomized trial comparing percutaneous vertebroplasty with conservative therapy. Am J Med 2003;114(4):257-265

11 Klazen CA, Lohle PN, de Vries J, et al. Vertebroplasty versus conservative treatment in acute osteoporotic vertebral compression fractures (Vertos II): an open-label randomised trial. Lancet 2010;376(9746): 1085-1092

12 Yuan WH, Hsu HC, Lai KL. Vertebroplasty and balloon kyphoplasty versus conservative treatment for osteoporotic vertebral compression fractures: A meta-analysis. Medicine (Baltimore) 2016;95(31):e4491

13 Trout AT, Kallmes DF, Kaufmann TJ. New fractures after vertebroplasty: adjacent fractures occur significantly sooner. AJNR Am J Neuroradiol 2006;27(1):217-223

14 Lindsay R, Silverman SL, Cooper C, et al. Risk of new vertebral fracture in the year following a fracture. JAMA 2001;285(3):320-323

15 Lindsay R, Burge RT, Strauss DM. One year outcomes and costs following a vertebral fracture. Osteoporos Int 2005;16(1):78-85

16 Xie W, Jin D, Wan C, et al. The incidence of new vertebral fractures following vertebral augmentation: a meta-analysis of randomized controlled trials. Medicine (Baltimore) 2015;94(37):e1532

17 Polikeit A, Nolte LP, Ferguson SJ. The effect of cement augmentation on the load transfer in an osteoporotic functional spinal unit: finite-element analysis. Spine 2003;28(10):991-996

18 Zhang H, Xu C, Zhang T, Gao Z, Zhang T. Does percutaneous vertebroplasty or balloon kyphoplasty for osteoporotic vertebral compression fractures increase the incidence of new vertebral fractures? A meta-analysis. Pain Physician 2017;20(1):E13-E28

19 Zhang Z, Fan J, Ding Q, Wu M, Yin G. Risk factors for new osteoporotic vertebral compression fractures after vertebroplasty: a systematic review and meta-analysis. J Spinal Disord Tech 2013;26(4):E150-E157 Review

20 Saracen A, Kotwica Z. Complications of percutaneous vertebroplasty: an analysis of 1100 procedures performed in 616 patients. Medicine (Baltimore) 2016;95(24):e3850

21 Yang W, Song J, Liang M, Cui H, Chen H, Yang J. Functional outcomes and new vertebral fractures in percutaneous vertebroplasty and conservative treatment of acute symptomatic osteoporotic vertebral compression fractures. World Neurosurg 2019;131:e346-e352

22 Wang B, Guo H, Yuan L, Huang D, Zhang H, Hao D. A prospective randomized controlled study comparing the pain relief in patients with osteoporotic vertebral compression fractures with the use of vertebroplasty or facet blocking. Eur Spine J 2016;25(11):3486-3494

23 Zuo XH, Zhu XP, Bao HG, et al. Network meta-analysis of percutaneous vertebroplasty, percutaneous kyphoplasty, nerve block, and conservative treatment for nonsurgery options of acute/subacute and chronic osteoporotic vertebral compression fractures (OVCFs) in short-term and long-term effects. Medicine (Baltimore) 2018;97(29):e11544 